\title{
5-Aminolevulinic Acid Improves Water Content and Reduces Skin Wrinkling
}

\author{
Yuna Naraoka1, Ailing Hu², Takuji Yamaguchi ${ }^{2}$, Norio Saga ${ }^{3}$, Hiroyuki Kobayashi ${ }^{1,2}$ \\ ${ }^{1}$ Department of Hospital Administration, Graduate School of Medicine, Juntendo University, Tokyo, Japan \\ ${ }^{2}$ Center for Advanced Kampo Medicine and Clinical Research, Graduate School of Medicine, Juntendo University, Tokyo, Japan \\ ${ }^{3}$ Institute of Sports Science \& Medicine, Teikyo University, Tokyo, Japan \\ Email: ynaraoka@juntendo.ac.jp
}

How to cite this paper: Naraoka, Y., Hu, A.L., Yamaguchi, T., Saga, N. and Kobayashi, H. (2020) 5-Aminolevulinic Acid Improves Water Content and Reduces Skin Wrinkling. Health, 12, 709-716. https://doi.org/10.4236/health.2020.127052

Received: June 3, 2020

Accepted: June 29, 2020

Published: July 2, 2020

Copyright (c) 2020 by author(s) and Scientific Research Publishing Inc. This work is licensed under the Creative Commons Attribution International License (CC BY 4.0).

http://creativecommons.org/licenses/by/4.0/

\section{(cc) (i) Open Access}

\begin{abstract}
5-Aminolevulinic acid (ALA) is a natural amino acid that is used as a raw material for the biosynthesis of red-blood-cell hemoglobin in humans and animals. ALA is the subject of research in a wide range of human health care fields, including skin care and medicine. This study investigated whether and to what extent cosmetics containing ALA (skin lotions and creams) improved facial-skin condition in women. As such, a double-blind controlled experiment was conducted among 45 women aged $47.0 \pm 1.72$ years. Participants were divided into two intervention groups, including those who used cosmetics containing ALA (C-ALA, $\mathrm{n}=22$ ) and those who used cosmetics without ALA (W-ALA, $n=23$ ). Specifically, participants were instructed to use their assigned cosmetics twice per day (morning and evening) after washing their faces before each application. Inner skin condition and skin-water content were evaluated via VISIA-Evolution and Multi Display Devices at baseline and after four and eight weeks of use. The experiment lasted for a total of eight weeks. A baseline comparison at week four showed that skin moisture content increased significantly for the C-ALA group $(\mathrm{p}=$ 0.021). Further, wrinkling significantly decreased at week four among participants in the C-ALA group who were evaluated as having many wrinkles at baseline $(p=0.034)$. These findings suggest that cosmetics containing ALA have moisturizing effects and reduce wrinkling caused by dryness.
\end{abstract}

\section{Keywords}

5-Aminolevulinic Acid, Wrinkle, Cosmetics, Facial Skin

\section{Introduction}

5-Aminolevulinic acid (ALA) is the first product in the porphyrin synthesis 
pathway. It is a natural amino acid found in animals and plants that is synthesized from glycine and succinyl CoA via mitochondrial aminolevulinic acid synthase and converted to protoporphyrin humans [1] [2]. Once accumulated in mitochondria, protoporphyrin forms heme by binding to iron. Heme is a material for hemoglobin, but can also be converted into cytochromes that work in the inner mitochondrial membrane, where they are essential for mitochondrial energy production [3]. Many common foods contain ALA, including spinach, green peppers, tomatoes, bananas, and ground beef [4]. However, the amount of ALA produced in the body decreases with age; the mitochondrial function also declines [5], thus causing fatigue and aging.

It has been clarified that oral intake of ALA improved the activity of the complex of the mitochondrial electron transport system in the liver and the production of ATP in mice. Improving mitochondrial function also improves basal metabolism, which leads to the improvement of various diseases [6]. Rodriguez and colleagues found that ALA has the effect of reducing fasting blood glucose in prediabetic patients and improving glucose metabolism during the glucose tolerance test [7]. Moreover, Sato and colleagues found that ALA improves lipid metabolism in rats that have been fed a high-fat diet, causing visceral fat accumulation [8]. Thus, ALA is expected to be effective in preventing lifestyle-related diseases caused by an abnormal metabolic endocrine system.

ALA has also been applied in the aesthetic medical field. For instance, because protoporphyrin emits red fluorescence, dermatological researchers have used ALA as a photosensitizer in photodynamic therapy to treat skin cancer [9]. Several previous studies have also suggested that topical photodynamic therapy mediated by ALA is effective for repairing photoaging tissues [10] [11] [12] [13]. There are also some promising new cosmetic applications for ALA beyond treatments designed to improve skin dryness and wrinkling. However, ALA has not been studied as a cosmetic product, these effects are not yet fully understood. As such, this study examined whether and to what extent cosmetics containing ALA improved wrinkling and helped retain moisture in the facial skin.

\section{Materials and Methods}

\subsection{Participants}

A total of 45 women were recruited through study advertisements placed at Juntendo University in Tokyo, Japan. The specific inclusion criteria were as follows: healthy women aged 20 - 69 years and with good skin condition. Exclusion criteria were as follows: persons who use external medications that may influence study results or those deemed unsuitable for participation per the discretion of a medical doctor.

After pre-study screening, 45 total eligible women were selected for participation. Average participant age was $47.0 \pm 1.72$ years [mean \pm standard error (SE)]. All were informed about the possible risks and discomforts involved in the experiment prior to giving their written informed consent to participate. His study's design was approved by the Juntendo University Ethical Review Board. Further, 
all procedures were conducted in accordance with the principles of the amended Declaration of Helsinki.

\subsection{Test Cosmetics}

Cosmetics containing ALA as the main active ingredient (i.e., lotion and cream) were supplied by SBI (SBI ALApromo CO., LTD., Tokyo, Japan). The products also included hydrolyzed hyaluronic acid, hyaluronic acid sodium, pentetic acid, red algae, tocopherol, olive fruit oil, soybean oil, and jojoba seed oil as moisturizing ingredients. Because participants were instructed to use the cosmetics twice per day by manufacturer's instruction, the estimated amount of total daily received ALA phosphate was $31 \mathrm{ng}$ per individual. Participants used both the lotion and cream.

\subsection{Procedures}

This study conducted a double-blind experiment involving an eight-week intervention. Participants were divided to ensure equal age distributions and then placed into two intervention groups, including one for cosmetics containing ALA (C-ALA) $(n=22)$ and one for cosmetics without ALA (W-ALA) $(n=23)$ (the control group). Both groups were instructed to use their respective cosmetics twice per day. The subjects washed their faces as usual and were prohibited from using beauty liquids, lotions, and creams other than the test products. Facial skin condition was measured at Juntendo University before the intervention and at both four and eight-week intervals after first application. This study was conducted from July 2018 to May 2019 in Juntendo University.

\subsection{Skin Condition Assessment}

Participants washed their faces to remove the cosmetics immediately after arriving at the measurement location. Skin condition was measured after resting for 20 minutes in a room set to a temperature of $24^{\circ} \mathrm{C}$. Skin evaluations included the following items: UV stains, brown spots, texture, pores, redness, and porphyrin used with VISIA ${ }^{\oplus}$ Evolution (VISIA-Evo, Integral Corporation, Tokyo). Further, facial skin under the eyes was measured for moisture content using via Multi Display Devices ${ }^{\circledast}$ MDD4 (MDD4, Integral Corporation, Tokyo).

\subsection{Statistical Analysis}

This study conducted statistical tests using SPSS ver. 26.0 (SPSS, IBM) (all significance levels were set to $\mathrm{p}<0.05)$. Skin condition was assessed using the Wilcoxon signed rank test. All data were expressed as means \pm standard errors unless otherwise specified.

\section{Results}

\subsection{The Effects of ALA on Moisture Contents in Facial Skin}

Compared to baseline, results showed that skin moisture content significantly 
increased in the C-ALA group after eight weeks $(p=0.028)$. Moreover, moisture contents of the facial skin significantly increased $(p=0.031)$ in the C-ALA group based on both the four and eight-week measurements (Figure 1). On the other hand, no significant changes were found in the W-ALA group throughout the study period. These findings suggest that prolonged use of cosmetics containing ALA may enhance moisture retention in the skin.

\subsection{Effects on Wrinkling}

No significant intergroup differences were found in regard to UV stains, brown stains, texture, pore condition, redness, or porphyrin. However, some participants' stains had apparently lightened after 8 weeks (Figure 2). On the other hand, participants in the C-ALA group who were assessed as having more than 30 wrinkles pre-intervention experienced significant reductions after four weeks $(\mathrm{p}=0.034)$ (Figure 3$)$. On the other hand, no significant intergroup differences were found based on eight-week measurements.

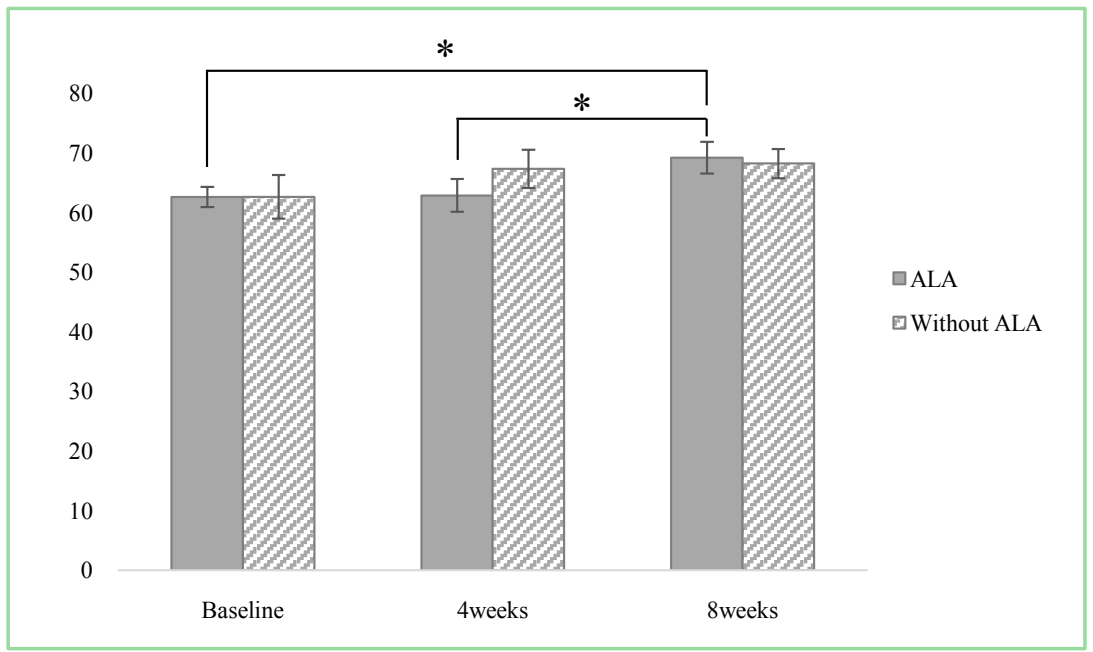

Figure 1. The effects of ALA on moisture content. Measurements before using, base line; measurements after four weeks; measurements after eight weeks. Values represent mean \pm SE.

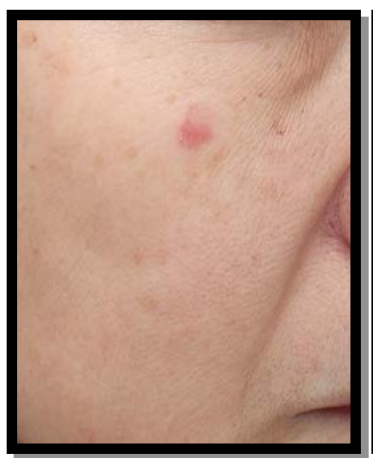

Baseline

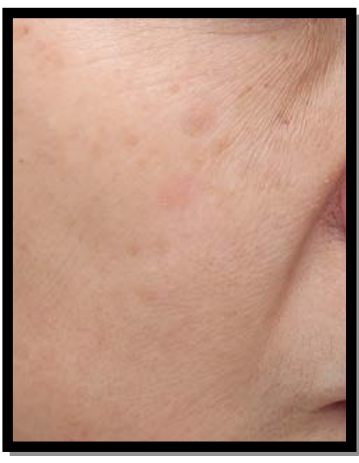

4 weeks

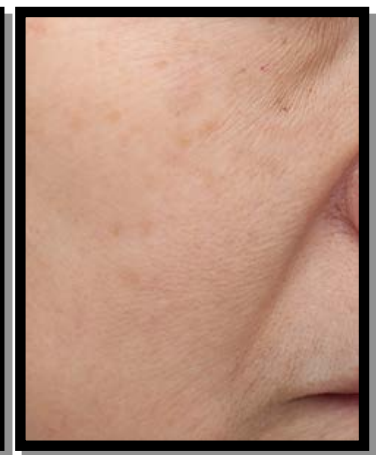

8 weeks

Figure 2. Changes in appearance of stains over 8 weeks. Pictures were taken using VISIA $^{\oplus}$ Evolution before using (baseline), after four weeks, and after eight weeks. 


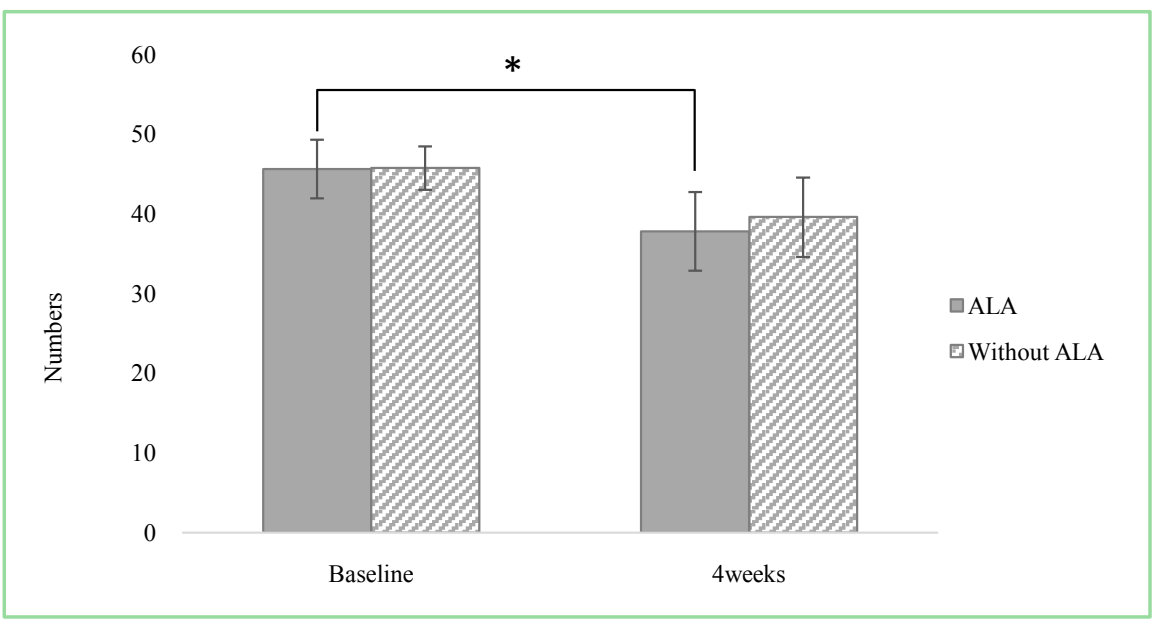

Figure 3. The effects of ALA on facial skin wrinkling. Measurements before using, baseline; measurements after four weeks. Values represent mean \pm SE.

\section{Discussion}

Moisture content is an important factor for skin smoothness. That is, the skin loses moisture as the amount of water decreases due to aging and/or lifestyle issues; this may result in wrinkling and the loss of skin flexibility [14]. Further, the mitochondrial function is activated via the binding of eight ALA molecules and one iron molecule [1]. This not only produces metabolic water, but also activates fibroblasts. Previous research has shown that an admixture of ALA and iron ion produces a significant effect on hair growth in mice when compared with iron ion alone [15]. In this study, mitochondrial activity appears to have been promoted because the tested cosmetics included both iron (as pentetate) and ALA. Moreover, the skin may have retained moisture because fibroblasts activated via mitochondrial production produced moisturizing amino acids, such as collagen and hyaluronic acid.

Human skin contains a stratum corneum, epidermal cells, and basal cells from the outside. When applying active ingredients (e.g., through cosmetics) along the surface of the skin, there is a problem in which the stratum corneum acts a barrier. Many believe that it is highly difficult for materials attached to the surface of the skin to reach basal cells situated below the $7-10$ rows of epidermal cells in order to reach the deeper dermis. Research indicates that a molecular weight of under $500 \mathrm{Da}$ is required to pass through the stratum corneum [16]. Notably, it is thus thought that the common beauty ingredients of collagen and hyaluronic acid cannot penetrate the stratum corneum due to their higher molecular weights. Specifically, the molecular weights of collagen and hyaluronic acid are around 100,000 and 1,000,000 $\mathrm{Da}$ or more, respectively, while ALA is much lower at 131.1 $\mathrm{Da}$ [17] [18]. For this reason, ALA can better promote turnover of the basal skin layer. By extension, the C-ALA group in this study used ingredients that penetrated to the basal layer, thus increasing and maintaining water contents. 
Since ALA can be used to increase water retention, it was expected that participants with large numbers of wrinkles caused by dryness would experience reductions. While the C-ALA group showed improvements at four weeks, the lack of differences found at eight weeks was likely due to low seasonal humidity, which stressed the skin. Further, no significant improvements were observed among participants who were assessed as having a few wrinkles pre-intervention. In this regard, since mitochondrial promotion activated via ALA improves skin moisture contents, it is presumed that fine wrinkles caused by dryness became less noticeable. Specifically, mitochondrial activation results in activated fibroblasts and turnover, which may reduce the number of wrinkles. However, the limitations of our study need to be considered in the interpretation of results. It is difficult to evaluate the effects of cosmetics alone because the effects of temperature, humidity, and ultraviolet rays due to the seasons and the effects of air conditioners are inevitable. Furthermore, it should be taken into consideration that skin conditions also depend on sleep time, stress, and diet.

\section{Conclusion}

In conclusion, cosmetics containing ALA phosphate (31 ng/day) improves skin moisture contents and reduces wrinkles when using the cosmetics twice per day.

\section{Acknowledgements}

The authors thank all participants and professional staff as well as the company members of SBI ALApromo Co., Ltd. This study was funded by SBI ALApromo Co., Ltd., Tokyo, Japan.

\section{Conflicts of Interest}

The authors declare no conflicts of interest regarding the publication of this paper.

\section{References}

[1] Hendry, G.A. and Jones, O.T. (1980) Haems and Chlorophylls: Comparison of Function and Formation. Journal of Medical Genetics, 17, 1-14. https://doi.org/10.1136/jmg.17.1.1

[2] Hara, T., Koda, A., Nozawa, N., Ota, U., Kondo, H., Nakagawa, H., Kamiya, A., Miyashita, K., Itoh, H., Nakajima, M. and Tanaka, T. (2016) Combination of 5-Aminolevulinic Acid and Ferrous Ion Reduces Plasma Glucose and Hemoglobin A1c Levels in Zucker Diabetic Fatty Rats. FEBS Open Bio, 6, 515-528. https://doi.org/10.1002/2211-5463.12048

[3] Castro, C.E. (1980) Mechanisms of Reaction of Hemeproteins with Oxygen and Hydrogen Peroxide in the Oxidation of Organic Substrates. Pharmacology \& The rapeutics, 10, 171-189. https://doi.org/10.1016/0163-7258(80)90080-7

[4] Perez, M.H., Rodriguez, B.L., Shintani, T.T., Watanabe, K., Miyanari, S. and Harrigan, R.C. (2013) 5-Aminolevulinic Acid (5-ALA): Analysis of Preclinical and Safety Literature. Food and Nutrition Sciences, 4, 1009-1013.

https://doi.org/10.4236/fns.2013.410131 
[5] Hayashi, J., Ohta, S., Kagawa, Y., Kondo, H., Kaneda, H., Yonekawa, H., Takai, D. and Miyabayashi, S. (1994) Nuclear But Not Mitochondrial Genome Involvement in Human Age-Related Mitochondrial Dysfunction. Functional Integrity of Mitochondrial DNA from Aged Subjects. The Journal of Biological Chemistry, 269, 6878-6883.

[6] Ogura, S., Maruyama, K., Hagiya, Y., Sugiyama, Y., Tsuchiya, K., Takahashi, K., et al. (2011) The Effect of 5-Aminolevulinic Acid on Cytochrome C Oxidase Activity in Mouse Liver. BMC Research Notes, 17, 66.

https://doi.org/10.1186/1756-0500-4-66

[7] Rodriguez, B.L., Curb, J.D., Davis, J., Shintani, T., Perez, M.H., Apau-Ludlum, N., et al. (2012) Use of the Dietary Supplement 5-Aminiolevulinic Acid (5-ALA) and Its Relationship with Glucose Levels and Hemoglobin A1C among Individuals with Prediabetes. Clinical and Translational Science, 5, 314-320.

https://doi.org/10.1111/j.1752-8062.2012.00421.x

[8] Sato, T., Yasuzawa, T., Uesaka, A., Izumi, Y., Kamiya, A., Tsuchiya, K., Kobayashi, Y., Kuwahata, M. and Kido, Y. (2014) Type 2 Diabetic Conditions in Otsuka Long-Evans Tokushima Fatty Rats Are Ameliorated by 5-Aminolevulinic Acid. $\mathrm{Nu}$ trition Research, 34, 544-551. https://doi.org/10.1016/j.nutres.2014.04.013

[9] Hayashi, M., Fukuhara, H., Inoue, K., Shuin, T., Hagiya, Y., Nakajima, M., Tanaka, T. and Ogura, S-I. (2015) The Effect of Iron Ion on the Specificity of Photodynamic Therapy with 5-Aminolevulinic Acid. PLoS ONE, 10, e0122351. https://doi.org/10.1371/journal.pone.0122351

[10] Ruiz-Rodriguez, R., Lopezm, L., Candelas, D. and Pedraz, J. (2008) Photorejuvenation Using Topical 5-Methyl Aminolevulinate and Red Light. Journal of Drugs in Dermatology, 7, 633-637.

[11] Touma, D., Yaar, M., Whitehead, S., Konnikov, N. and Gilchrest, B.A. (2004) A Trial of Short Incubation, Broad-Area Photodynamic Therapy for Facial Actinic Keratoses and Diffuse Photodamage. Archives of Dermatological Research, 140, 33-40. https://doi.org/10.1001/archderm.140.1.33

[12] Marmur, E.S., Phelps, R. and Goldberg, D.J. (2005) Ultrastructural Changes Seen after ALA-IPL Photorejuvenation: A Pilot Study. Journal of Cosmetic and Laser Therapy, 7, 21-24. https://doi.org/10.1080/147641700510037725

[13] Orringer, J.S., Hammerberg, C., Hamilton, T., Johnson, T.M., Kang, S., Sachs, D.L., et al. (2008) Molecular Effects of Photodynamic Therapy for Photoaging. Archives of Dermatology, 144, 1296-1302. https://doi.org/10.1001/archderm.144.10.1296

[14] Wilhelm, K.P., Cua, A.B. and Maibach, H.I. (1991) Skin Aging. Effect on Transepidermal Water Loss, Stratum Corneum Hydration, Skin Surface pH, and Casual Sebum Content. Archives of Dermatology, 127, 1806-1809. https://doi.org/10.1001/archderm.1991.04520010052006

[15] Morokuma, Y., Yamazaki, M., Maeda, T., Yoshino, I., Ishizuka, M., Tanaka, T., Ito, Y. and Tsuboi, R. (2008) Hair Growth Stimulatory Effect by a Combination of 5-Aminolevulinicacid and Iron Ion. International Journal of Dermatology, 47, 1298-1303. https://doi.org/10.1111/j.1365-4632.2008.03783.x

[16] Bos, J.D. and Meinardi, M.M. (2000) The 500 Dalton Rule for the Skin Penetration of Chemical Compounds and Drugs. Experimental Dermatology, 9, 165-169. https://doi.org/10.1034/j.1600-0625.2000.009003165.x

[17] Park, J.M., Jeong, K.H., Bae, M.I., Lee, S.J., Kim, N.I. and Shin, M.K. (2016) Fractional Radiofrequency Combined with Sonophoresis to Facilitate Skin Penetration of 5-Aminolevulinic Acid. Lasers in Medical Science, 31, 113-118. 
https://doi.org/10.1007/s10103-015-1835-1

[18] Sorushanova, A., Delgado, L.M., Wu, Z., Shologu, N., Kshirsagar, A., Raghunath, R., et al. (2019) The Collagen Suprafamily: From Biosynthesis to Advanced Biomaterial Development. Advanced Materials, 31, e1801651.

https://doi.org/10.1002/adma.201801651 